
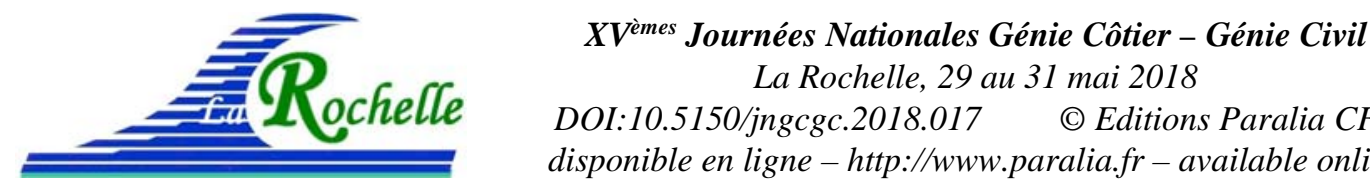

DOI:10.5150/jngcgc.2018.017～(C) Editions Paralia CFL

disponible en ligne - http://www.paralia.fr - available online

\title{
Conséquences de l'implantation des ouvrages de protection sur la frange côtière de Bordj El Kiffan (Algérie)
}

\section{Cherif AOUDJ ${ }^{1,2}$, Ismahane KADRI ${ }^{1}$, Rabah BELKESSA ${ }^{1,2}$ Abdelkarim MEZHOUD ${ }^{1,2}$, Fouzia HOUMA BACHARI ${ }^{1,2}$, Abla HANTAL ${ }^{1}$}

1. Ecole Nationale Supérieure des Sciences de la Mer et de l'Aménagement du Littoral, ENSSMAL, Bois des Cars, B.P. 19 Dely Ibrahim, 16320, Algérie. cherifaoudj@gmail.com

2. Laboratoire de l'Écosystème Marin et Littoral, EcoSys, Chemin du complexe de Sidi Fredj, Staoueli 16096, Algérie.

\section{Résumé :}

L'anthropisation des rivages accélèrent les tendances d'évolutions ce qui mènent à d'autres interventions de l'Homme en implantant des ouvrages durs de protection qui peuvent avoir d'autres impacts et même accélérer les taux d'érosion (SAMAT, 2007).

La zone côtière de Bordj El Kiffan située dans la partie centrale de la baie d'Alger, a connu une artificialisation de son rivage traduite par la forte urbanisation depuis 1980. Cela a causé le recul remarquable du linéaire côtier. Pour y remédier, la solution préconisée par le laboratoire d'études maritimes est la combinaison de plusieurs méthodes de protection (voir figure 1).

Dans le but de connaitre et de déterminer les conséquences de l'implantation de ces ouvrages de protection, une étude d'évolution historique du trait de côte a été menée avec des SIG comme moyen de suivi, accompagnée des études hydrodynamique et sédimentaire de la partie marine de la zone d'étude tout en s'appuyant sur la modélisation numérique par le modèle couplé de Mike 21. Un déséquilibre sédimentaire considérable s'est enclenché de part et d'autre de la baie d'Alger.

Mots clés : Ouvrages de protection, ArcGis, Dynamique sédimentaire, Sédimentologie, SIG, Trait de côte, Mike 21, Modélisation numérique.

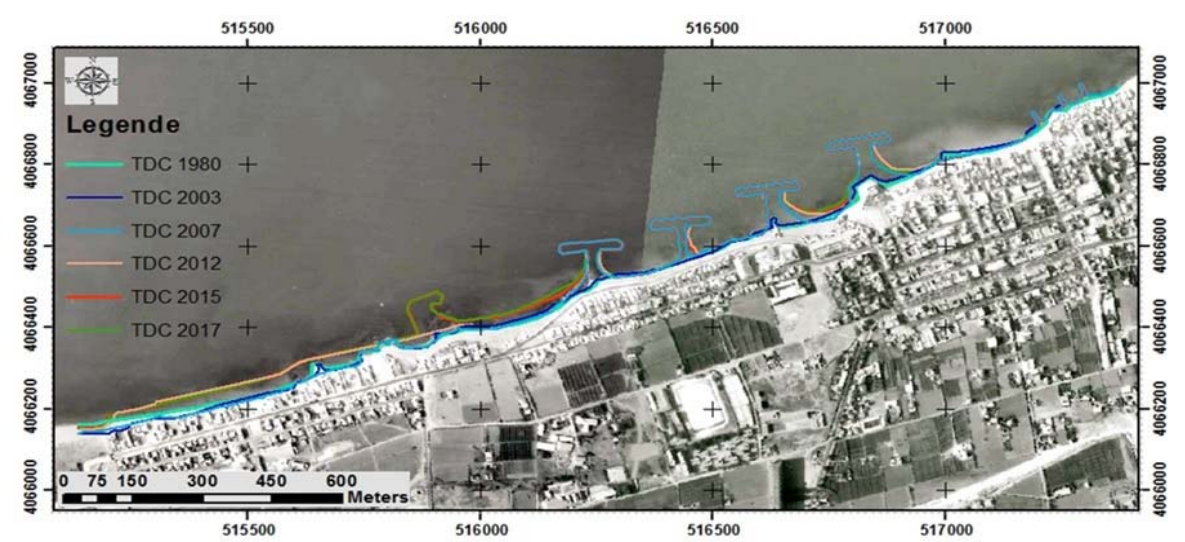

Figure 1. Évolutions de la ligne de rivage entre 1980 et 2017. 


\section{Thème 2 - Dynamique sédimentaire}

\section{Introduction}

Notre cas d'étude est la frange côtière de Bordj El Kiffan, située dans la partie centrale de la baie d'Alger (voir figure 2) s'étalant sur $2 \mathrm{~km}$, où certains ouvrages de protection ont été mis en place.

Il est nécessaire de comprendre la dynamique globale de la zone d'étude, pour ce faire, nous avons étudié son évolution dans une échelle à moyen terme, durant la période de 1980 à 2017, permettant de percevoir l'impact des ouvrages de protection qui y existent sur la variation de la ligne e rivage.

De plus, une analyse sédimentologique et une étude des champs de courants induits par la houle et le transport sédimentaire ont été établies afin de mieux cerner et comprendre le fonctionnement de notre frange côtière.

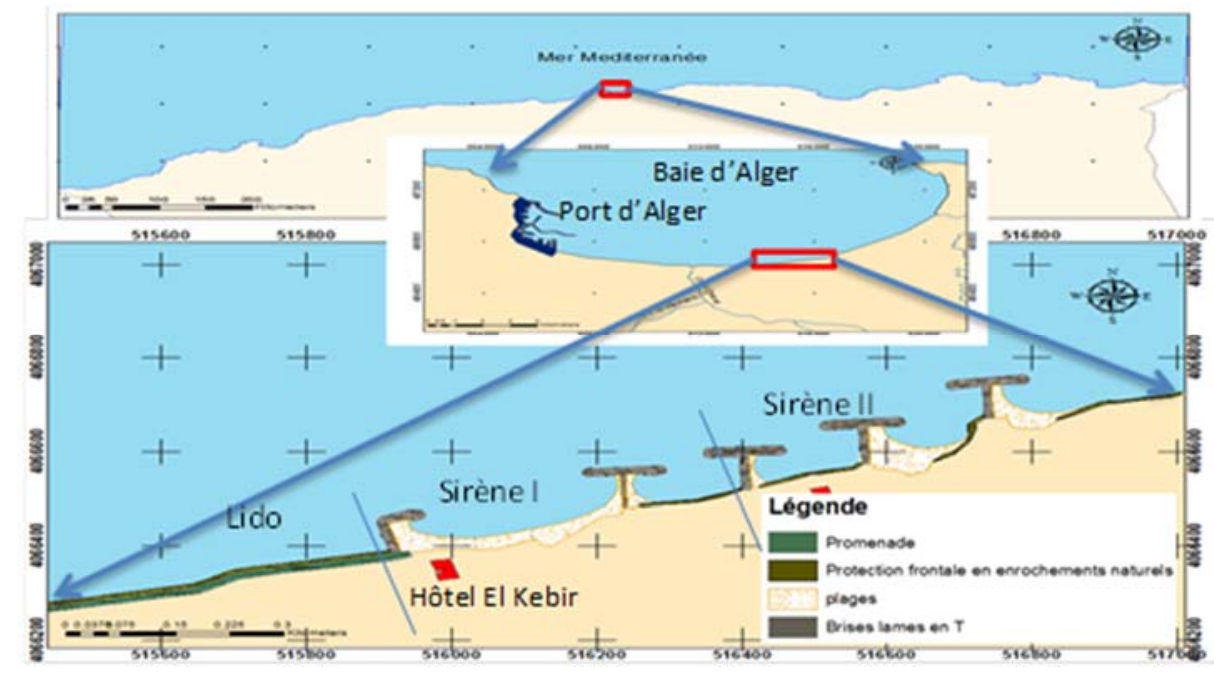

Figure 2. Carte de la localisation de la zone d'étude.

\section{Cinématique côtière}

\subsection{Outils et procédure}

Nous avons procédé à l'aide de l'analyse diachronique des photographies aériennes datant respectivement de 1980, 2003 et 2007, d'une orthophoto de 2012 et d'un levé sur terrain réalisé par le biais du GPS de type GARMIN effectué en 2017 (voir tableau1).

Tableau 1. Données utilisées pour déterminer l'évolution du trait de côte

\begin{tabular}{lllll}
\hline Date & Type de données & Source & Échelle & Résolution \\
\hline $\mathbf{0 8 / 1 9 8 0}$ & Photos aériennes $n^{\circ} 24$ et 26 & INCT & $1 / 10000$ & $1 \mathrm{~m}$ \\
$\mathbf{2 9} / \mathbf{0 5} / \mathbf{2 0 0 3}$ & Photos aériennes $n^{\circ}$ 28, 104 et 106 & $I N C T$ & $1 / 10000$ & $1 \mathrm{~m}$ \\
$\mathbf{2 2 / 1 2 / 2 0 0 7}$ & Photos aériennes $n^{\circ} 1437,1439$ et 1441 & $I N C T$ & $1 / 4000$ & $42 \mathrm{~cm}$ \\
$\mathbf{2 4 / 0 2 / 2 0 1 2}$ & Ortho photographie $n^{\circ} 600$ & $I N C T$ & $1 / 20000$ & $12 \mathrm{~cm}$ \\
$\mathbf{2 6 / 5 / 2 0 1 5}$ & Image satellitaire & Google Earth & $1 / 7000$ & $15 \mathrm{~cm}$ \\
$\mathbf{1 8 / 0 4 / 2 0 1 7}$ & Levé GPS & Levé terrain & $/$ & $/$ \\
\hline
\end{tabular}




\section{XVèmes Journées Nationales Génie Côtier - Génie Civil \\ La Rochelle, 29 au 31 mai 2018}

\subsubsection{Orthorectification et géoréférencement des photographies aériennes}

La rectification des photographies aériennes a permis de les superposer à l'orthophoto de 2012 pour déterminer le changement de la position du trait de côte.

Une fois les points de contrôle choisis, nous avons procédé à la rectification proprement dite des photos en utilisant le logiciel ArcGIS 10.2.

Le logiciel utilise les points de contrôle lors de cette rectification et procède à une transformation qui modifie les photos de telle sorte à optimiser la superposition.

La transformation choisie est celle dite du "Spline" de $1^{\text {er }}$ ordre. Le nombre minimal de points d'appuis peut être calculé de la façon suivante (SAMAT, 2007) :

$$
\mathrm{N}=(\mathrm{T}+1)(\mathrm{T}+2) / 2
$$

Où : $\quad \mathrm{N}$ : le nombre minimal d'amers nécessaire

$\mathrm{T}:$ l'ordre de la transformation

Le logiciel ne génère pas d'erreur résiduelle pour ce type de transformation.

Une fois les photographies aériennes rectifiées, les déformations sont fortement atténuées et l'image est géoréférencée. L'association des photographies d'une même compagne permet d'obtenir une image complète du site pour cette date.

\subsubsection{Digitalisation des différents traits de côte}

La digitalisation des traits de côtes a été faite à l'aide du module ArcMap du logiciel ArcGis 10.2, où une nouvelle base de données personnalisée a été créée afin de pouvoir l'utiliser ultérieurement dans l'extension Digital Shoreline Analysis System (DSAS) pour calculer les statistiques d'évolution des traits de côte.

\subsubsection{Calcul des erreurs induites par le traitement des données des traits de côte}

Les différents outils et méthodes utilisés induisent une erreur significative qui affecte la précision des données générées. Il est donc très important de calculer leur marge d'erreur et d'en tenir compte lors de l'appréciation des taux de changement du trait de côte et de leur utilisation ultérieure. Les sources d'erreur dont nous avons tenu compte sont les suivantes :

a. La précision des photos $E_{p}$

Elle correspond à la résolution de la photo. (ROBICHAUD, et al., 2012)

b. L'erreur due à la rectification $E_{r}$

Le plus souvent, on utilise l'erreur résiduelle générée par le logiciel de rectification qui est calculée à partir de la qualité de la superposition des points de contrôle selon la méthode de transformation utilisée (ROBICHAUD, et al., 2012).

c. L'erreur de digitalisation $E_{d}$

C'est l'erreur due au positionnement du trait de côte selon les repères choisis. Difficile à établir précisément, on utilise souvent une valeur arbitraire, mais raisonnable (ROBICHAUD, et al., 2012). Dans ce travail, elle est jugée égale à $\pm 5 \mathrm{~m}$ pour les photos de 1980 et $2003, \pm 2 \mathrm{~m}$ pour la photo de 2007 et $\pm 1 \mathrm{~m}$ pour l'orthophoto de 2012. 


\section{Thème 2 - Dynamique sédimentaire}

d. L'erreur due aux variations saisonnières $E_{s}$

C'est l'erreur due aux fluctuations saisonnières du rivage.

Les différences des positions saisonnières sont calculées sur les traits de côte d'hiver et d'été de 2007 et de 2012. Cette erreur est estimée à $\pm 3 \mathrm{~m}$

e. L'erreur globale

Elle est définie comme la racine carrée de la somme des carrées des erreurs. Elle est calculée en utilisant :

$$
E_{g}= \pm \sqrt[2]{E_{p}^{2}+E_{r}^{2}+E_{d}^{2}+E_{s}^{2}}
$$

Les erreurs sont donc estimées en (m) et résumées dans le tableau suivant :

Tableau 2. Estimation des erreurs induites lors du traitement des données des TDC.

\begin{tabular}{llllll}
\hline Date & $\begin{array}{l}\text { Erreur } \\
\text { résiduelle } \\
\text { RMS }\end{array}$ & $\begin{array}{l}\text { Erreur due } \\
\text { à la rectification } \\
\text { (choix des amers) }\end{array}$ & $\begin{array}{l}\text { Erreur de } \\
\text { digitalisation }\end{array}$ & $\begin{array}{l}\text { Résolution ou } \\
\text { précision de } \\
\text { l'orthophotoplan }\end{array}$ & $\begin{array}{l}\text { Erreur } \\
\text { globale } \\
(\mathrm{m})\end{array}$ \\
\hline $21 / 08 / 1980$ & 0 & 1 & 2 & 0.12 & 3.12 \\
$29 / 05 / 2003$ & 0 & 1 & 2 & 0.12 & 3.12 \\
$22 / 12 / 2007$ & 0 & 0.5 & 1 & 0.12 & 1.62 \\
$24 / 02 / 2012$ & 0 & 0.25 & 0.25 & 0.12 & 0.62 \\
$26 / 5 / 2015$ & 0 & 0.5 & 1 & 0.12 & 1.62 \\
\hline
\end{tabular}

2.1.4 Utilisation de l'extension DSAS pour l'analyse de l'évolution du trait de côte

Les mesures entre les traits ont été faites à l'aide de l'extension Digital Shoreline Analysis System (DSAS) spécialement conçu pour ce genre de travail et compatible avec le logiciel ArcGIS.

Les taux de déplacement annuels ont été calculés sur près de 250 transects ayant une longueur de $250 \mathrm{~m}$ et distants de 10 mètres tout le long du littoral.

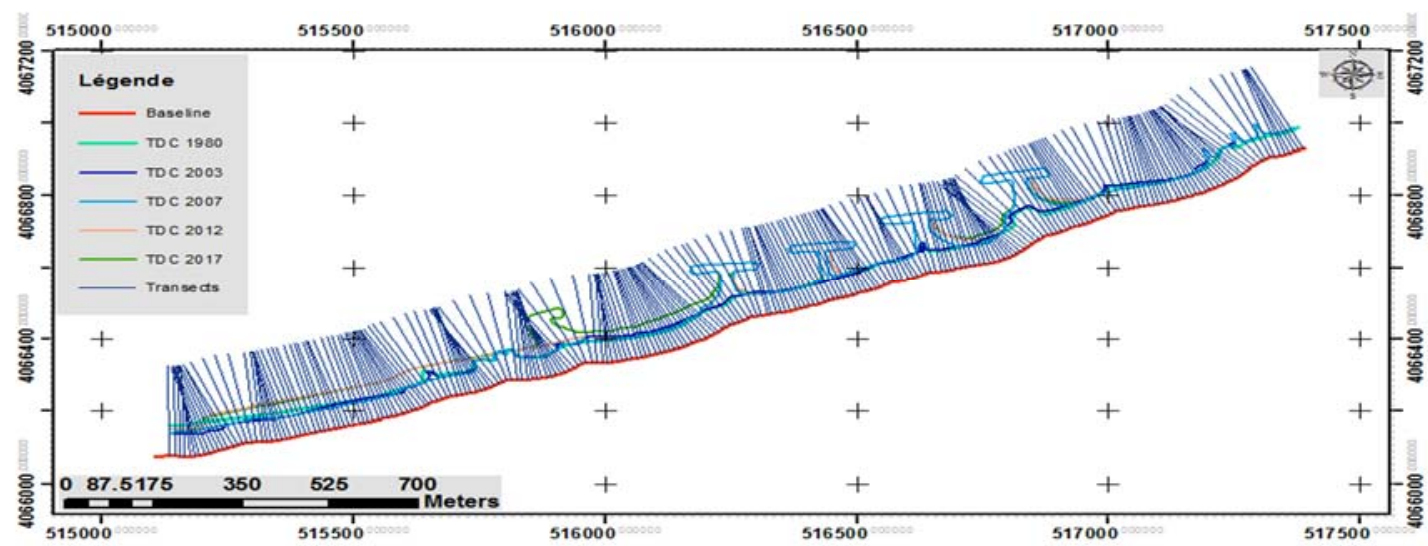

Figure 3. Génération des transects dans la zone d'étude à l'aide de l'extension DSAS. 


\section{XVèmes Journées Nationales Génie Côtier - Génie Civil \\ La Rochelle, 29 au 31 mai 2018}

\subsection{Résultats et discussion}

\subsection{1 Évolution du linéaire côtier entre 1980 et 2003}

Cette période est marquée par une accrétion moyenne de $0.24 \mathrm{~m} / \mathrm{an}$ dans la partie Est. Par contre dans la partie Ouest, l'érosion a pris l'avantage sur l'accumulation avec un recul moyen estimé à $-0.12 \mathrm{~m} / \mathrm{an}$. Ces évolutions de la côte sont d'une part, le résultat de la manifestation des forçages hydrodynamiques et d'autre part, l'intervention du facteur humain par la forte urbanisation du rivage et de son anthropisation.

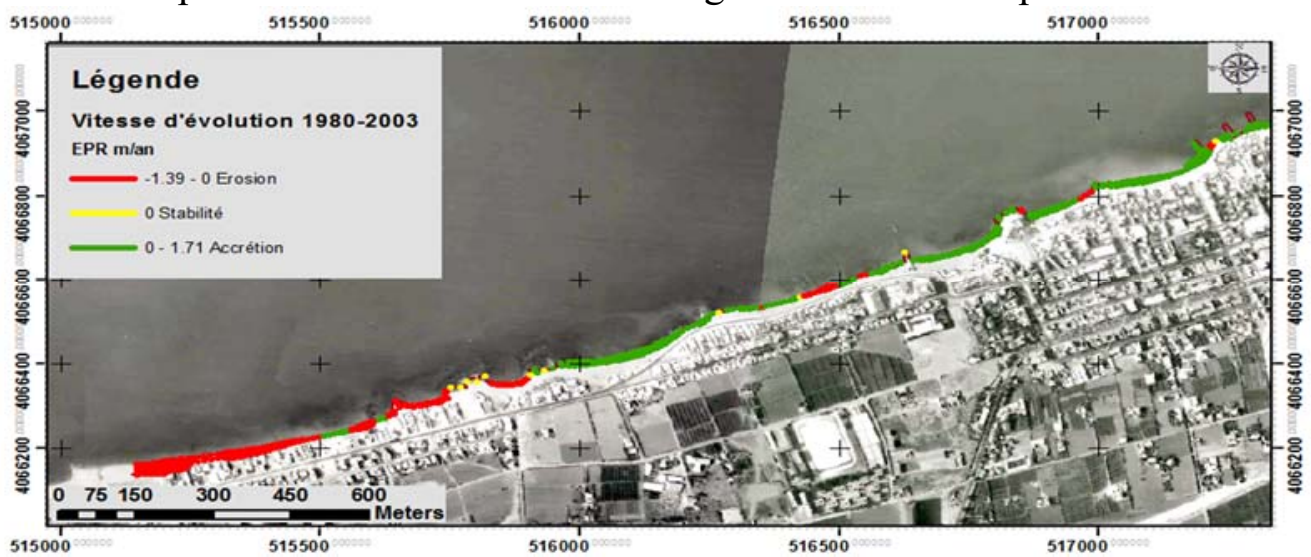

Figure 4. Vitesse d'évolution du trait de côte entre 1980 et 2003.

\subsection{2 Évolution du linéaire côtier entre 2003 et 2007}

Durant une période de 4 ans, notre zone d'étude a été marquée par une alternance de zone d'érosion et d'accrétion. L'érosion a été déclenchée au niveau du secteur Est et a été accentuée au niveau du secteur Ouest avec un taux moyen de $-1.17 \mathrm{~m} / \mathrm{an}$. Nous remarquons que les zones d'accumulation se situent en amont des épis avec une évolution moyenne de $2.73 \mathrm{~m} / \mathrm{an}$. Ces bouleversements à court terme sont dus à la combinaison de deux facteurs naturels et artificiels, par l'hydrodynamisme et par l'implantation de quatre épis en $\mathrm{T}$ qui favorisent l'érosion en aval et la progradation en amont de la dérive littorale.

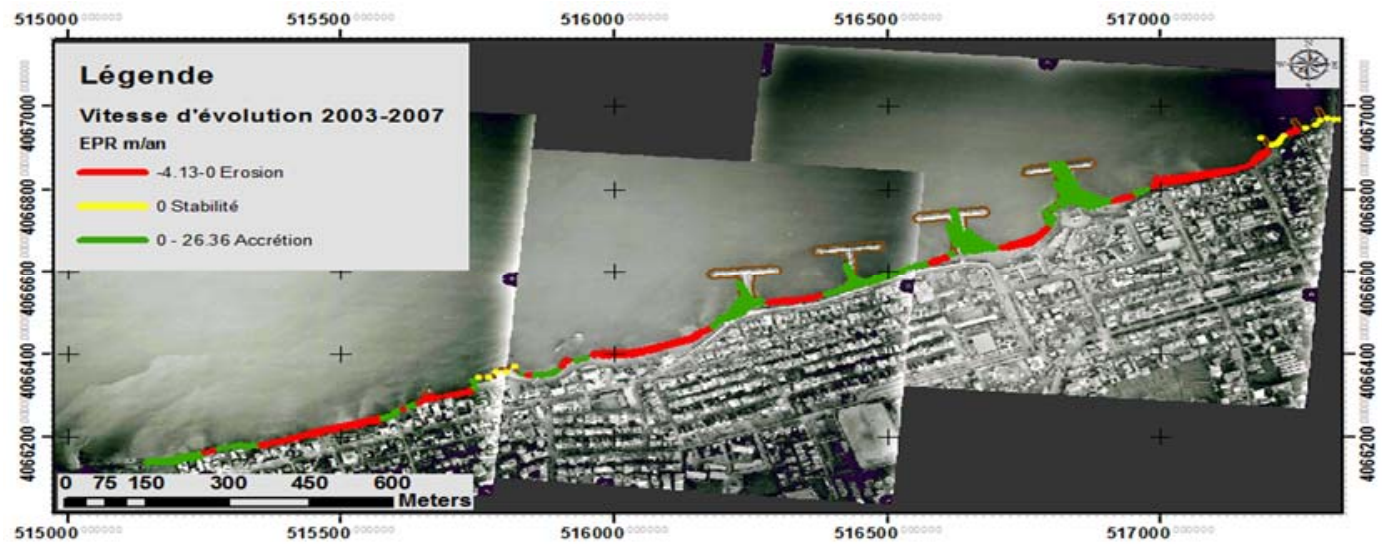

Figure 5. Vitesse d'évolution du trait de côte entre 2003 et 2007. 


\section{Thème 2 - Dynamique sédimentaire}

\subsection{3 Évolution du linéaire côtier entre 2007 et 2012}

De 2007 à 2012, les taux d'érosion ont fortement été atténués à une moyenne de -0.26 $\mathrm{m} / \mathrm{an}$. Nous remarquons la poursuite de l'engraissement surtout au niveau du secteur Ouest avec une moyenne de $5.98 \mathrm{~m} / \mathrm{an}$.

L'engraissement spectaculaire dans le secteur Ouest est engendré par le début des travaux de l'aménagement d'une promenade et une plage artificielle au niveau de Lido.

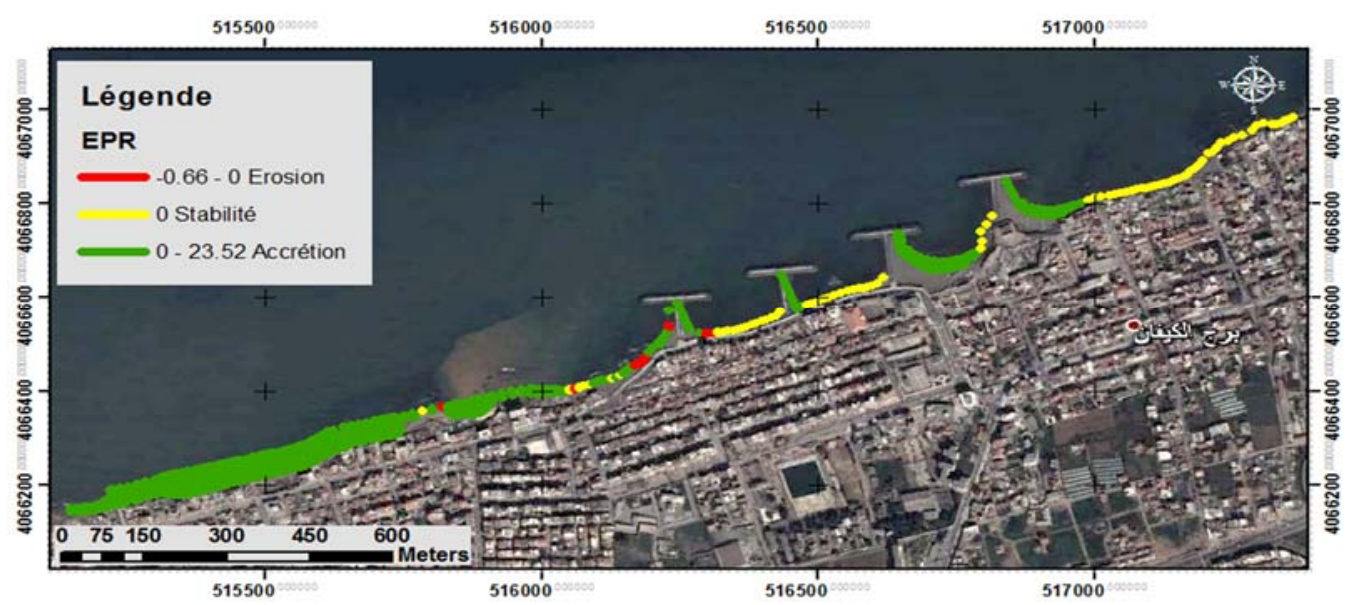

Figure 6. Vitesse d'évolution du trait de côte entre 2007 et 2012.

\subsection{4 Évolution du linéaire côtier entre 2012 et 2017}

Durant une période de 5 ans, l'érosion s'est déclenchée à nouveau dans le secteur Est en amont du dernier brise-lames et au niveau du Lido avec une vitesse moyenne de -0.72 $\mathrm{m} / \mathrm{an}$. La progradation au niveau du secteur Ouest continue avec une moyenne de 3.45 $\mathrm{m} /$ an. La poursuite de l'accumulation dans la plage Lido est artificielle et est causée par un rechargement artificiel ainsi que la mise en place d'un épi en L pour le maintenir.

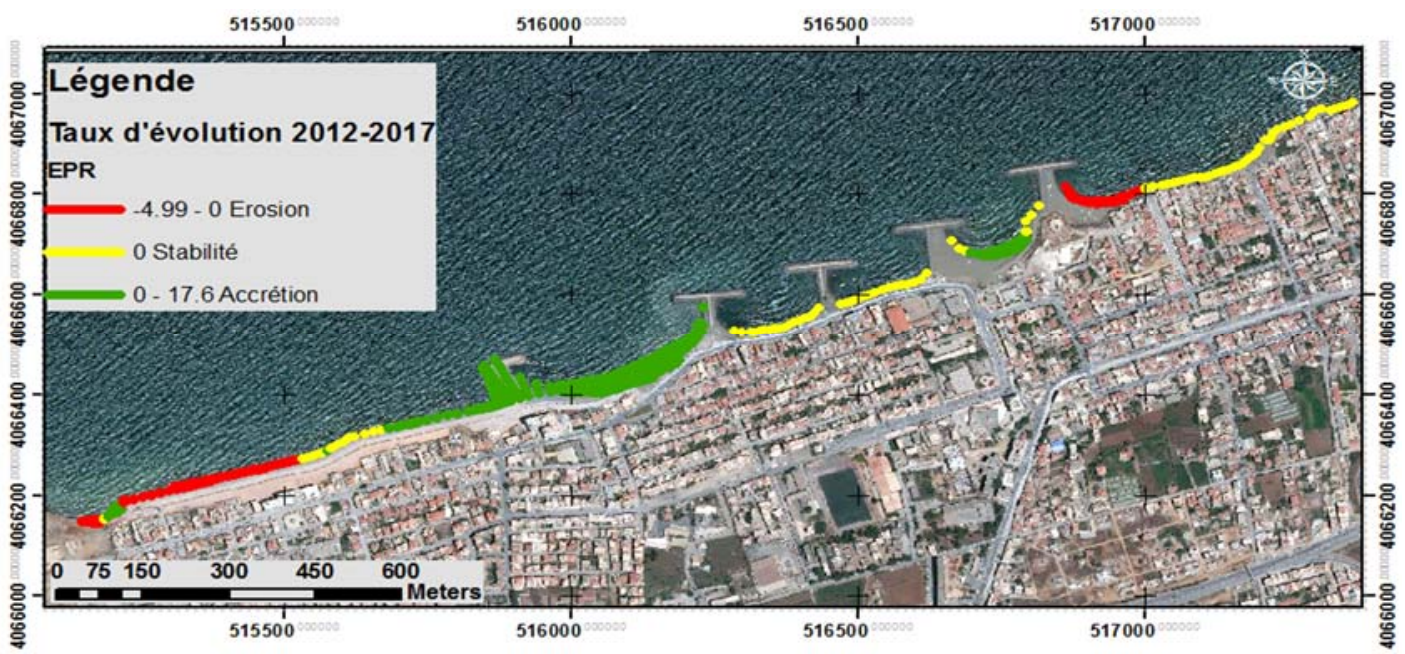

Figure 7. Vitesse d'évolution du trait de côte entre 2012 et 2017. 


\section{XVèmes Journées Nationales Génie Côtier - Génie Civil \\ La Rochelle, 29 au 31 mai 2018}

\section{3. Étude sédimentologique}

\subsection{Méthodologie suivie}

Dans le but de connaitre la nature et la répartition des sédiments sous-marins qui caractérisent notre zone d'étude, nous avons récolté 18 échantillons choisis aléatoirement lors de notre sortie en mer en utilisant une benne preneuse de type "Van Veen" à différentes profondeurs, la profondeur maximale était de $-11 \mathrm{~m}$. De plus 11 échantillons ont été prélevé à la côte. Ensuite, les échantillons obtenus ont été destinés à une analyse granulométrique au laboratoire.
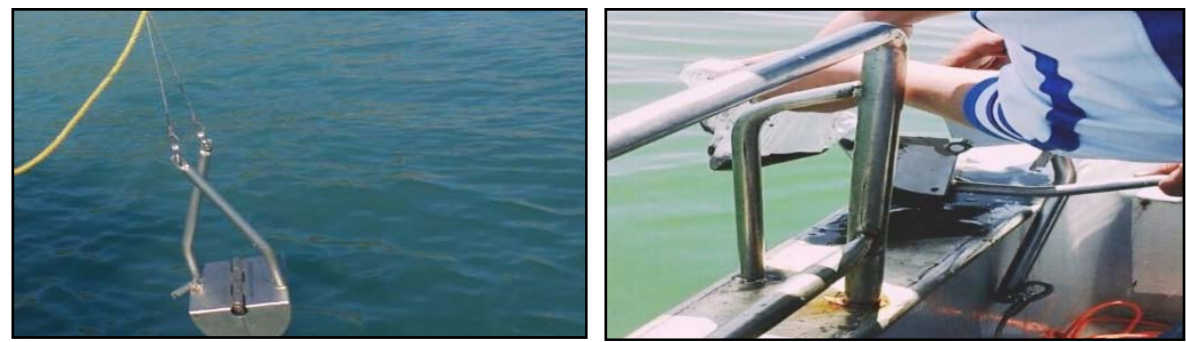

Figure 8. Prélèvement des échantillons à l'aide de la benne Van Veen.

\subsection{Résultats de l'analyse sédimentologique}

\subsubsection{La fraction fine}

La répartition de la fraction fine montre de faibles teneurs à la côte qui augmentent en allant vers le large, elles varient entre 0 et $7 \%$ sur l'ensemble du site. Cette répartition révèle la présence d'une énergie importante mettant en perpétuel remaniement les sédiments.

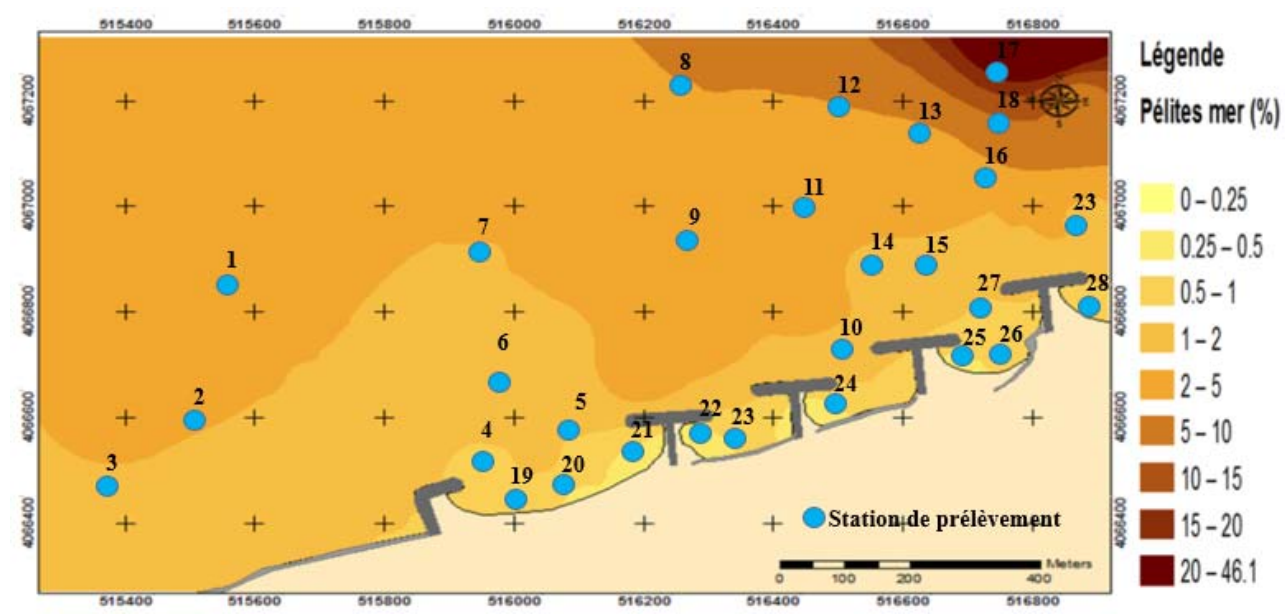

Figure 9. Répartition des pélites dans la plage sous-marine. 


\section{Thème 2 - Dynamique sédimentaire}

\subsubsection{La médiane}

La répartition de la médiane présente une diminution de la taille du grain moyen de la côte vers le large en partant de $970 \mu \mathrm{m}$ à $63 \mu \mathrm{m}$ environ donc du grossier vers le plus fin, tel qu'elle est représentée dans la figure 10.

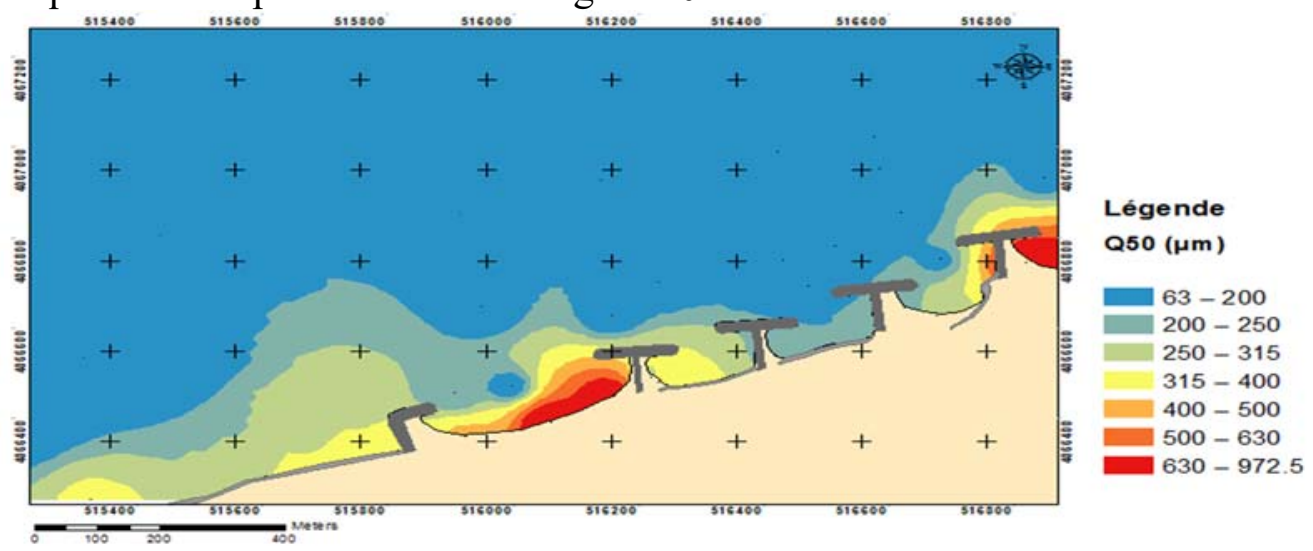

Figure 10. Répartition de la médiane dans la plage sous-marine.

\section{4. Étude hydrodynamique}

\section{1 $\underline{\text { Simulation des champs de courant et du transport }}$}

\subsubsection{Préparation des données d'entrée au modèle}

a. La bathymétrie du modèle sous forme d'un maillage avec l'extension ".mesh"

b. Les données de la houle : hauteur significative, période et direction :

Les houles utilisées pour cette étude sont celles de la période de $8 \mathrm{~s}$ et de direction N20 car elles sont les plus fréquentes et les plus énergétiques, voir tableau 3.

c. La table des sédiments générée à partir de la boite à outils de Mike 21 "Mike 21 toolbox"

Un diamètre moyen D50 de $290 \mu \mathrm{m}$ et un écart type $\sigma \mathrm{g}$ de $318 \mu \mathrm{m}$ sont retenus pour notre modèle.

Tableau 3. Caractéristiques de la houle retenues pour le module SW

\begin{tabular}{llll}
\hline \multicolumn{4}{c}{ Direction de la houle au large } \\
\hline Période & $N 320^{\circ}$ & $N 360^{\circ}$ & $N 20^{\circ}$ \\
\hline $8 s$ & $H s=2,54 \mathrm{~m}$ & $H s=2,47 \mathrm{~m}$ & $H s=2,56 \mathrm{~m}$ \\
\hline
\end{tabular}

\subsubsection{Réglage du modèle}

Les étapes sont : Création d'un espace de travail "CoupledModelFM.mfm"; Réglage de la durée de la simulation; Choix des modules: HD, ST et SW; Paramétrage des modules : données initiales, conditions aux limites, ...; Choix des fichiers de sortie : Vitesse du courant, capacité du transport ...; Lancer la simulation. 


\section{XVèmes Journées Nationales Génie Côtier - Génie Civil \\ La Rochelle, 29 au 31 mai 2018}

\subsection{Résultats de la simulation}

Nous constatons que les houles de direction N20 créent un courant de dérive avec des vitesses de l'ordre de $1.65 \mathrm{~m} / \mathrm{s}$. Ces courants ont une capacité de transport solide de l'ordre de 0.00112 à $0.00112 \mathrm{~m}^{3} / \mathrm{s} / \mathrm{m}$.

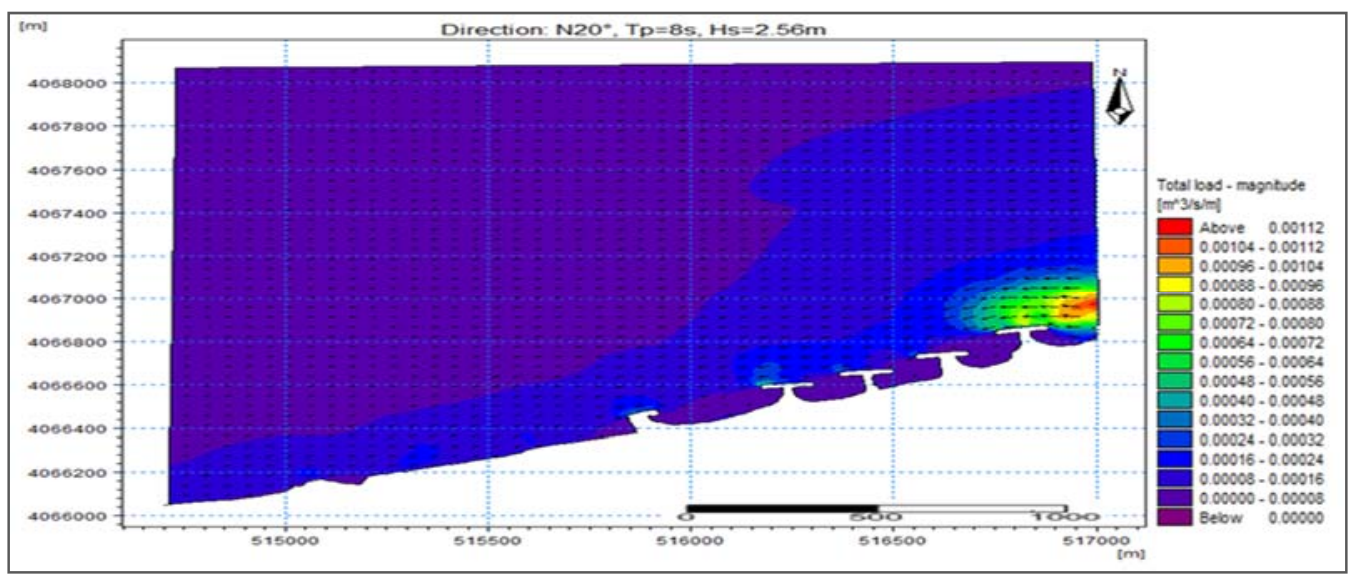

Figure 11. Champs de courant induits par la houle du secteur $\mathrm{N20}, \mathrm{Tp}=8 \mathrm{~s}$ et $\mathrm{Hs}=2.56 \mathrm{~m}$.

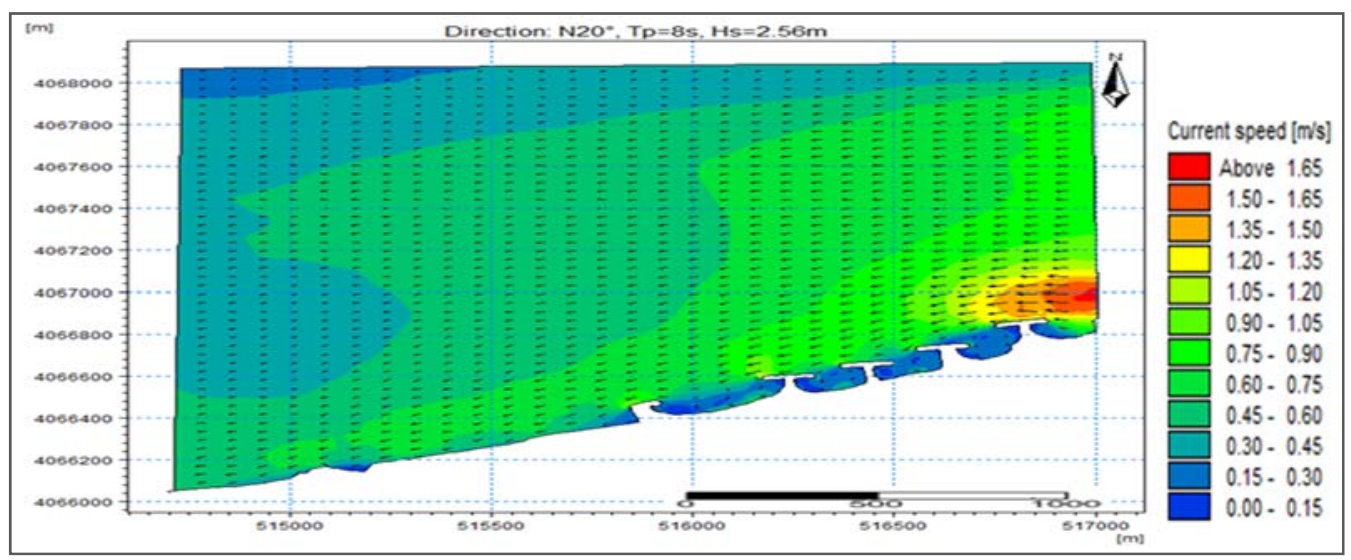

Figure 12. Capacité de transport solide par la houle du : Secteur N20, $\mathrm{Tp}=8 \mathrm{~s}$ et $\mathrm{Hs}=2.56 \mathrm{~m}$.

\section{Conclusions}

La construction des habitations le long la frange côtière de Bordj El Kiffan à partir de 1980 a favorisé le déclenchement du phénomène d'érosion.

La mise en place de différents ouvrages de protection (Brise-lames en $\mathrm{T}$ et $\mathrm{L}$, cordons d'enrochement, promenade et rechargement artificiel) au niveau du linéaire côtier de la zone a induit une instabilité remarquable de ce dernier: à savoir de forts taux d'accrétion et de faibles taux d'érosion où l'accrétion a pris l'avantage sur le recul. 


\section{Thème 2 - Dynamique sédimentaire}

La répartition de la médiane montre une dominance du sable fin au large de la zone d'étude et du sable grossier à l'intérieur des bassins des brise-lames.

La simulation numérique des champs de courants et du transport sédimentaires montrent que les houles les plus énergétiques du Nord-Est engendrent des courants avec des vitesses de l'ordre de $1.65 \mathrm{~m} / \mathrm{s}$ et un transport sédimentaire traduit par des courants de dérive littorale avec la dominance d'Est en Ouest, des courants tourbillonnaires à l'intérieur des bassins de brise-lames ainsi qu'un faible courant de retour. Ce qui a engendré un freinage donc, un stockage sédimentaire qui à la base avait comme destination la partie Est de la baie d'Alger d'où un déséquilibre voire, une érosion dans certaines plages voisines.

Remerciements : Nos remerciements s'adressent à l'ensemble de notre équipe de recherche, ainsi qu'à tous ceux qui ont organisé les XIV ème Journées Nationales Génie Côtier - Génie Civil. En particulier, Professeur Daniel LEVACHER.

\section{Références bibliographiques}

AOUDJ C., BOUAZIZ T. (2014). Fonctionnement et dynamique morpho-sédimentaires du littoral Est de Béjaïa. Mémoire d'ingéniorat, ENSSMAL, Alger, pp 81-94.

AOUDJ C., MEZHOUD A., GUERFI M., HEMDANE Y. (2017). Analyse des variations spatio-temporelles du littoral sableux: Est Béjaoui (Algérie). Conférences Méditerranéennes $\mathrm{CM}^{2}$, Split Croatie, pp 7-10. https://dx.doi.org/10.5150/cmcm.2017.002

BELKESSA R. (2004). Reconnaissance de sites de dépôts des produits de dragage portuaires sur le plateau et le littoral algériens (méthodologie et paramètres d'étude). contrat Ismal/Cnepru.

KADRI I. (2017). Impact de l'implantation des ouvrages de protection sur la frange côtière de Bordj El Kiffan. Mémoire d'ingéniorat, ENSSMAL, Alger, 44 p.

ROBICHAUD A., CHELBI M., SIMARD I. (2012). Erosion et infrastructures à risque à Sainte-Marie-Saint-Raphael Péninsule Acadienne, Nouveau-Brunswick. Université de Moncton [Rapport]. novembre $39 \mathrm{p}$.

SAMAT O. (2007). Effiacité et impacts des ouvrages en enrochement sur les plages microtidales. Le cas du Languedoc et du Delta du Rhone. [Thèse de doctorat en Géographie physique et humaine, aménagement, urbanisme]. s.1., France : Université d'Aix-Marseille I, 376 p. 